\title{
Spectroscopic Study of the Dimerization Process of Iron Protoporphyrin IX
}

\author{
K. Dziedzic-Kocurek ${ }^{a}$, H.J. Byrne ${ }^{b}$, A. Ś'Widerski ${ }^{c}$ AND J. StAneK ${ }^{a}$ \\ ${ }^{a}$ Marian Smoluchowski Institute of Physics, Jagiellonian University \\ Reymonta 4, 30-059 Kraków, Poland \\ ${ }^{b}$ Focas Institute, Dublin Institute of Technology, Kevin Street, Dublin 8, Ireland \\ ${ }^{c}$ Department of Biochemistry, Faculty of Horticulture, University of Agriculture in Kraków \\ al. 29 Listopada 54, 31-425 Kraków, Poland
}

\begin{abstract}
The commercial protoporphyrin IX, iron-ferriprotoporphyrin IX-chloride and synthesized iron porphyrin $\mu$-oxo-dimers were examined by UV/vis absorption and fluorescence, Fourier transformed infrared spectroscopy, resonance Raman, X-ray absorption, Mössbauer spectroscopy and SQUID. The evidence of Fe-O-Fe antiferromagnetic coupling concluded from SQUID and Mössbauer in the case of samples containing dimerized forms confirmed the presence of the oxo-bridges. In this paper the results of UV/vis, fluorescence, Fourier transform infrared FTIR and Raman spectroscopies are reported and discussed. The study is based on the comparison of the free-base protoporphyrin IX, Fe-PPIX-Cl and the synthesized dimerized specimen. The vibrational modes in two energy regions i.e. $330-650 \mathrm{~cm}^{-1}$ and $750-900 \mathrm{~cm}^{-1}$, reportedly characteristic of the existence of $\mathrm{Fe}-\mathrm{O}-\mathrm{Fe}$ bridges, are discussed. A significant photoluminescence emission, strongly Stokes shifted from the Soret band, absent in the protoporphyrin IX and the iron-ferriprotoporphyrin IX-chloride, is observed. The strong Stokes shift and the mismatch of the excitation spectrum to the Soret band suggest that is does not have origin in the de-excitation of the porphyrin moiety and that it could have origin in an Fe-O-Fe charge transfer state.
\end{abstract}

PACS numbers: 36.20.-r, 36.20.Kd, 36.20.Ng

\section{Introduction}

Metalloporphyrins, which contain $\mathrm{Fe}, \mathrm{Mg}, \mathrm{Mn}, \mathrm{Co}$, $\mathrm{Cu}$, Ni or Zn suitably nested between the four nitrogens of pyrrole rings play an important role in biological systems as functional groups of hemeproteins, oxidases, chlorophyls and cobalamins [1-3]. Porphyrin derivatives have also recently attracted attention because of potential clinical applications $[4,5]$. As photosensitizing agents they have proven, for example, to be very useful photosensitizers in the photodynamic therapy (PDT) of cancer $[6,7]$. Dimerization and oligomerization of ironpophyrins have been studied in various model systems for more than 30 years. However, the mechanism of interaction between the haem centers remains unclear, especially in the most important porphyrin IX. The most often investigated systems are $\mu$-oxo-bridged dimers of ironporphyrins. The schematic structure of the ironporphyrin molecule and one of its possible dimer forms is shown in Fig. 1.

Iron porphyrin $\mu$-oxo-dimers were synthesized according to the modified Adler procedure. The results of a study of the local dynamical properties of the iron atoms in the porphyrin monomers and $\mu$-oxo-dimers have been reported previously [8]. The strong antiferromagnetic behavior observed in both Mössbauer and SQUID measurements confirms a strong coupling between iron centers characteristic of dimerization. In this study the characterization of the material using $\mathrm{UV} /$ visible and mid

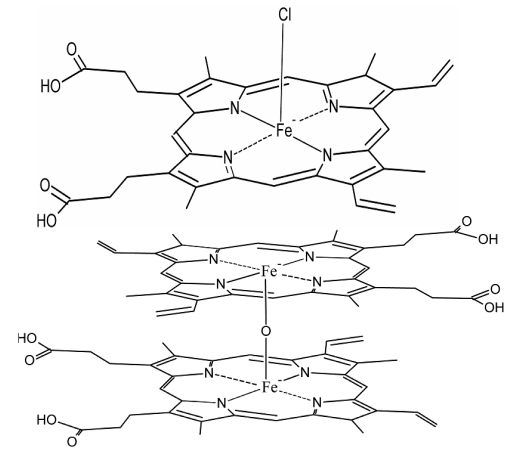

Fig. 1. Molecular structure of the iron porphyrin monomer (top) and iron porphyrin dimer (bottom).

infrared absorption, fluorescence, and Raman spectroscopies has been carried out. In all cases the spectra are referenced to those of the protoporphyrin and the metal monomer.

\section{Experimental}

Iron porphyrin dimers were prepared by insertion of iron atoms from ${ }^{57} \mathrm{FeCl}_{3}$ into protoporphyrin IX (PPIX) (Sigma) and finally precipitated from water- $N, N$-dimethylformamide (DMF) solution, according to the modified Adler procedure $[9,10]$.

According to some modifications during the preparation, final samples containing different amounts of the 
$\mu$-oxo dimers were obtained. For the final analysis, a sample of $(\mathrm{FeP})_{2} \mathrm{O}$ consisting of around $70 \%$ of the dimer as determined by SQUID and Mössbauer measurements was chosen [11]. The impurities within this sample consist of the ${ }^{57} \mathrm{Fe}-\mathrm{PPIX}-\mathrm{Cl}$ and trace amounts of PPIX.

This synthesized product $(\mathrm{FeP})_{2} \mathrm{O}$, the commercial PPIX (Sigma) and ferriprotoporphyrin IX chloride (Fe-PPIX-Cl) (Alfa Aesar) were tested by UV/vis and Fourier transform infrared (FTIR) absorption spectroscopy as well as by fluorescence and Raman spectroscopy. In the case of $\mathrm{UV} / \mathrm{vis}$ absorption and $\mathrm{flu}-$ orescence spectroscopy the samples were examined in dimethylformamide (DMF) solution, while in the FTIR and Raman spectroscopies as $\mathrm{KBr}$ pellets (1\% concentration).

$\mathrm{UV} /$ vis absorption spectra were taken with the use of the JASCO V-530 spectrometer with a spectral resolution of $2 \mathrm{~nm}$. The fluorescence spectra were taken by a Hitachi F-4500 spectrophotometer. The emission slit was $2.5 \mathrm{~nm}$ while the excitation slit was set for $2.5 \mathrm{~nm}$ or $5 \mathrm{~nm}$ in the case of fluorescence emission or excitation measurements, respectively.

The FTIR measurements were performed with a Bruker "Equinox 55" interferometer, modified to work under vacuum. System parameters: FIR range (10$700 \mathrm{~cm}^{-1}$ ), source: $\mathrm{Hg}$ lamp, detector: Si bolometer at $4.2 \mathrm{~K}$; mid infrared (MIR) range $\left(600-5000 \mathrm{~cm}^{-1}\right)$, source: globar (silicon carbide rod), detector: MCT. The spectral resolution was $4 \mathrm{~cm}^{-1}$ and the sample was a $1 \%$ by weight $\mathrm{KBr}$ pellet.

The Raman spectroscopy was carried out using an Instruments S.A. (Horiba Jobin-Yvon) Labram 1B operating at a wavelength of $514.5 \mathrm{~nm}$. The power at the sample was $\approx 10 \mathrm{~mW}$ and a $10 \times$ microscope objective was employed. To avoid photodegradation the powdered materials were mixed $(\approx 1 \%)$ with $\mathrm{KBr}$ and pressed into pellet form. Whereas samples were visibly degraded in their pure powder form, no degradation of the Raman signal was observable during prolonged exposure/measurement (minutes) and no visible degradation was observable microscopically in the $\mathrm{KBr}$ pellet form.

\section{Results and discussion}

$\mathrm{UV} /$ vis absorption spectroscopy was carried out in order to establish any signatures of electronic interaction between the porphyrin moiety and the bridging unit, and to identify the optimum excitation wavelengths for photoemission studies. Figure 2 shows the UV/vis absorption spectrum of PP-IX, Fe-PPIX-Cl, $(\mathrm{FeP})_{2} \mathrm{O}$. The spectra in all cases are dominated by the characteristic porphyrin Q-bands $(\approx 500-620 \mathrm{~nm})$ and Soret band $(\approx 400 \mathrm{~nm})$. The abrupt step at $\approx 400 \mathrm{~nm}$ is an artefact of the spectrometer. The Soret band is largely unchanged in the dimer products. The doublet Q-band splitting of the protoporphyrin is removed in the Fe-PPIX-Cl due to the increased symmetry in the metal environment. In the $(\mathrm{FeP})_{2} \mathrm{O}$ sample, these bands have become broadened probably due to the incomplete reaction. There is

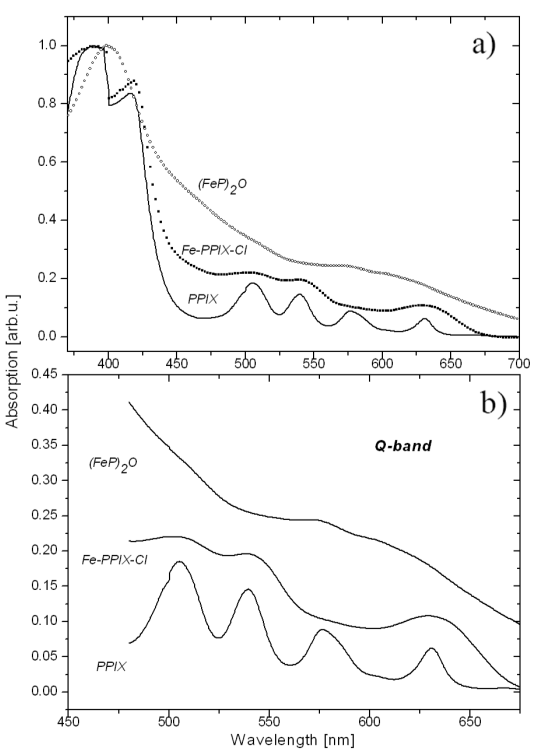

Fig. 2. (a) The UV/vis absorption spectra of all compounds (normalized at the Soret band for visual clarity). (b) The Q-band of the UV/vis of all compounds.

however no compelling evidence in the spectrum of the $(\mathrm{FeP})_{2} \mathrm{O}$ compound of specific signatures of the Fe-O-Fe bridge.

Figure 3 shows the FTIR spectra of the products compared with the Fe-PPIX-Cl monomer and PPIX. Of interest is the feature at $\approx 840 \mathrm{~cm}^{-1}$ which has previously been attributed to the asymmetric stretch of the Fe-O-Fe bridge [12-15]. The fact that both PPIX and Fe-PPIX-Cl exhibit absorption in this region is an indication that these vibrational modes originate from the porphyrin ring, however. The band at $\approx 840 \mathrm{~cm}^{-1}$ in PPIX is shifted to higher wave number in the Fe-PPIX$\mathrm{Cl}$ by $\approx 14 \mathrm{~cm}^{-1}$, consistent with a stiffening of the ring by replacement of the central hydrogens by the metal. The band is softened in the $(\mathrm{FeP})_{2} \mathrm{O}$ compound, which is indicative of an increased effective mass of the central metal. While dimerization softens this mode it cannot be simply attributed to the Fe-O-Fe bridge. The FTIR spectrum of the porphyrin PPIX is extremely rich in this region and although many studies have assigned the strong bands in this region to direct evidence of Fe-O-Fe bridging [16-19] no identifiable independent signature of such a bridge is evident in Fig. 3.

Upon excitation at $600 \mathrm{~nm}$, all compounds show characteristic porphyrin-like $S_{0}-S_{1}$ fluorescence emission features at $\approx 630 \mathrm{~nm}$. Excitation into the Soret band at $405 \mathrm{~nm}$ also gives rise to $S_{0}-S_{1}$ emission. While the PPIX and Fe-PPIX-Cl showed no indication of an $S_{2}-S_{0}$ emission, $(\mathrm{FeP})_{2} \mathrm{O}$ exhibits a notable emission at $\approx 480 \mathrm{~nm}$ (Fig. 4). Zinc porphyrins have been reported to exhibit $S_{2}-S_{0}$ emission although they are normally characterized by a small Stokes shift of $\approx 5 \mathrm{~nm}$ associated with the macromolecular ring relaxation $[20,21]$. The emission in Fig. 4 has a significantly larger Stokes shift of $\approx$ 


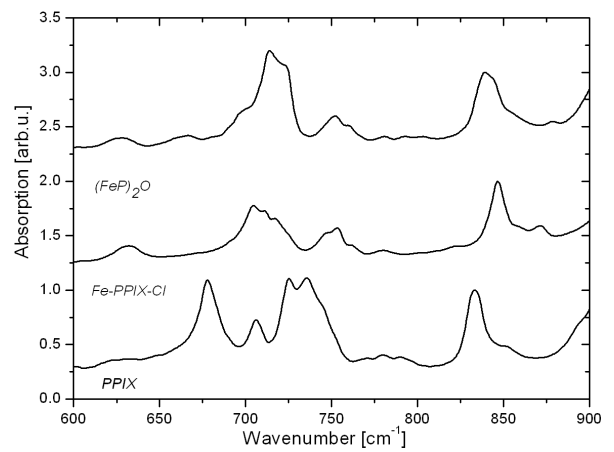

Fig. 3. The FTIR spectra of all compounds (normalized to the peak around $840 \mathrm{~cm}^{-1}$ ). The spectra are offset for clarity.

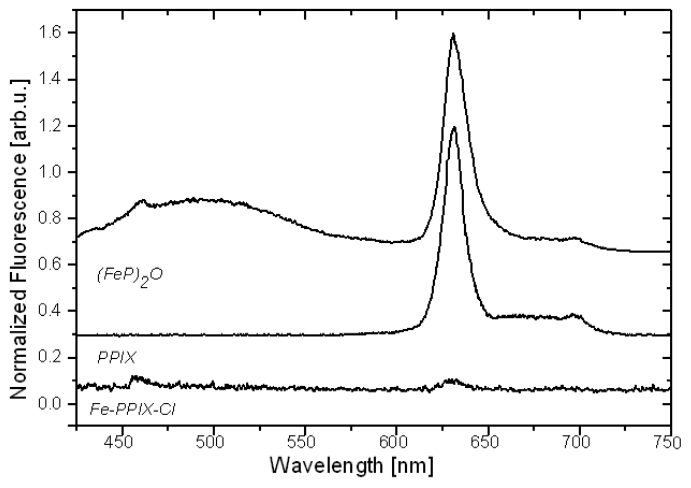

Fig. 4. The fluorescence spectra for all componds (excitation wavelength $405 \mathrm{~nm}$, normalized at $631 \mathrm{~nm}$ ). The spectra are offset for clarity.

$100 \mathrm{~nm}(\mathrm{eV})$ suggesting that it is not associated with the $\pi$-conjugated ring. Fe-O-Fe bridges have been reported to exhibit UV/vis absorbances at 360-380 nm which have been attributed to charge transfer states [15, 22-25]. Although they are not evident in Fig. 2, such an absorption could be present in the region of the Soret band. Figure 5 shows the excitation spectrum of the emission of

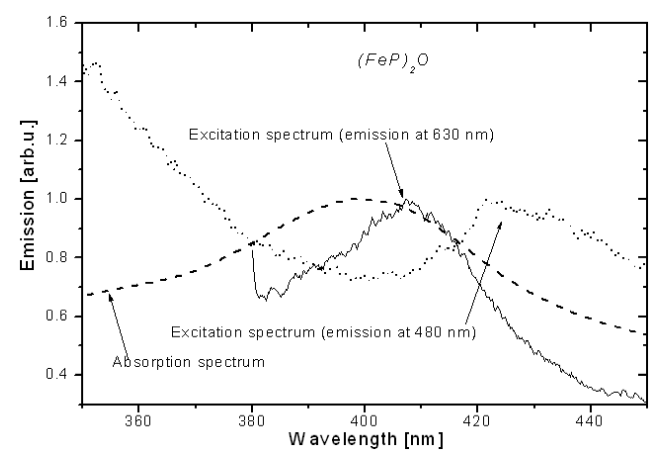

Fig. 5. Excitation spectra of $(\mathrm{FeP})_{2} \mathrm{O}$. The spectra are offset for clarity.
$(\mathrm{FeP})_{2} \mathrm{O}$ at $630 \mathrm{~nm}$ compared to that at $480 \mathrm{~nm}$. The emission at $480 \mathrm{~nm}$ is clearly of different origin than the porphyrin based emission at $630 \mathrm{~nm}$, whose excitation spectrum compares well with the porphyrin based Soret band emission.

The excitation spectrum has a maximum of $430 \mathrm{~nm}$, considerably shifted from both that of the $630 \mathrm{~nm}$ emission $(\approx 407 \mathrm{~nm})$ and the absorption spectrum, also shown in Fig. 5. The presence of this emission band, the associated Stokes shift and the relative positioning of the excitation maximum suggest that it is not associated with the porphyrin ring but rather with an $\mathrm{Fe}-\mathrm{O}-\mathrm{Fe}$ bridge charge transfer state.

The Raman spectra of the compounds recorded at $514.5 \mathrm{~nm}$ are shown in Fig. 6. Similar to the FTIR spectra of Fig. 3, they are rich in the fingerprint regions 400 $1700 \mathrm{~cm}^{-1}$. The complexity of the spectra makes it difficult to identify any features uniquely attributable to the dimer and consequently the Fe-O-Fe bridge. While the asymmetric stretch of bridged porphyrin dimers has been reported to lie at $\approx 850 \mathrm{~cm}^{-1}$, the symmetric stretch, which should be Raman active, has been reported to lie at $\approx 450 \mathrm{~cm}^{-1}[14,15,25-28]$. In the spectra of Fig. 6 , however, there is no evidence of characteristic features in this region. $(\mathrm{FeP})_{2} \mathrm{O}$ does however uniquely show a feature at $895 \mathrm{~cm}^{-1}$ which could be a candidate for the asymmetric stretch which may be swamped by the strong ring absorptions in the FTIR spectra. Previous studies have demonstrated that the symmetric stretch is weak compared to the overall compound spectrum and becomes prominent only upon resonant excitation at $\approx 400 \mathrm{~nm}$. Excitation is into the $\mathrm{Fe}-\mathrm{O}-\mathrm{Fe}$ bridge charge transfer state indicated above giving a clear signature of the Fe-O-Fe bridge. Such resonant excitation was not available for this study however and at $514.5 \mathrm{~nm}(\mathrm{FeP})_{2} \mathrm{O}$ shows significant absorption associated with the porphyrin implying that the ring modes are resonantly enhanced.

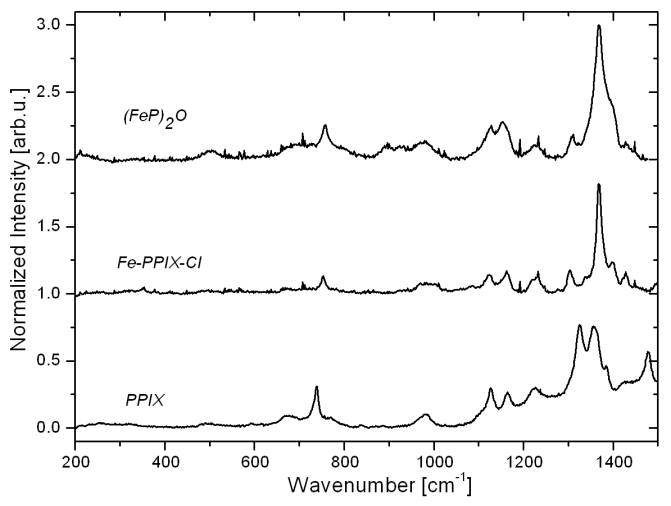

Fig. 6. Raman spectra of all compounds (normalized to the peak around $1630 \mathrm{~cm}^{-1}$ ). The spectra are offset for clarity.

\section{Summary and conclusion}

The spectroscopy of the porphyrin based species in all cases is dominated by the $\pi$-conjugated macrocycle. 
No unique signature of the dimer in $(\mathrm{FeP})_{2} \mathrm{O}$, akin to the magnetic signatures of SQUID and Mössbauer spectroscopy, has been identified although strong supporting evidence is provided.

The UV/vis absorption spectra of all compounds are dominated by the strongly absorbing $\pi$-conjugated ring. The FTIR spectra are very complex and shifts of porphyrin ring modes, consistent with dimerization, can be observed. Features often assigned in literature to Fe-O-Fe bridges clearly have origin in the porphyrin ring, however. It is possible that the bridge modes underlie the very much stronger ring modes. Fluorescence spectroscopy provides more compelling evidence for a bridge based charge transfer state. The $(\mathrm{FeP})_{2} \mathrm{O}$ compound shows a significant emission at $\approx 480 \mathrm{~nm}$, strongly Stokes shifted from the Soret band which has a significantly different excitation spectrum to the porphyrin emission, consistent with the presence of a charge transfer absorption feature in this region. Such an absorption feature in the UV/vis spectrum would be swamped by the strong Soret band, however. The Raman spectrum shows no evidence of the predicted Fe-O-Fe symmetric stretch mode at $\approx 450 \mathrm{~cm}^{-1}$ although the product $(\mathrm{FeP})_{2} \mathrm{O}$ exhibits a feature at $895 \mathrm{~cm}^{-1}$ which may be the asymmetric stretch which is swamped by the ring modes in the FTIR spectrum. The excitation wavelength employed is resonant with the ring absorptions and predictably the spectrum is dominated by the porphyrin based bands. Resonant excitation into the bridge charge transfer states at $\approx 400 \mathrm{~nm}$ should be employed to yield a clear signature of the dimer bridge.

\section{Acknowledgments}

This work has been supported by: HASYLAB-DESY, Hamburg, Germany under the contract RII3-CT-2004506008-DESY-D-II-20060195 EC，LNF-Frascati, Italy, TARI Contract Number RII3-CT-2004-506078-Proposal No. 44. FTIR spectra were recorded in the INFN-LNF Laboratory in Frascati. Katarzyna Dziedzic-Kocurek would like to thank Dr hab. Kvetoslava Burda - for an introduction to porphyrins, as well as discussions, suggestions and assistance during the FTIR measurements. Special acknowledgments are due to Mariangela Castelli Guidi and Massimo Picinini for their support and suggestions during the FTIR measurements. The authors would like to acknowledge Dr. Paweł Kaszycki for his professional help and suggestions during the fluorescence measurements. This collaboration is funded in part through the EU FP7 SSA DASIM.

\section{References}

[1] L.R. Milgrom, The Colours of Life - An Introduction to the Chemistry of Porphyrins and Related Compounds, Oxford University Press, New York 1997, p. 1.

[2] L. Stryer, Biochemistry, 4th ed. Wydawnictwo Naukowe PWN, Warszawa 2003, p. 153, 573, 683, 699 (in Polish).
[3] R.H. Holm, P. Kennepohl, E.I. Solomon, Chem. Rev. 96, 2239 (1996).

[4] J.W. Smalley, M.F. Thomas, A.J. Birss, R. Withnall, J. Silver, Biochem. J. 379, 833 (2004).

[5] A. Dorn, S.R. Vippagunta, H. Matile, C. Jaquet, J.L. Vennerstrom, R.G. Ridley, Biochem. Pharmacol. 55, 727 (1998).

[6] D. Zaak, R. Sroka, S. Stocker, K. Bise, M. Lein, M. Höppner, D. Frimberger, P. Schneede, O. Reich, M. Kriegmair, R. Knüchel, R. Baumgartner, A. Hofstetter, Urol. Int. 72, 196 (2004).

[7] R. Muschter, Curr. Urol. Rep. 4, 221 (2003).

[8] K. Dziedzic-Kocurek, J. Stanek, K. Burda, Hyperfine Interact., to be published.

[9] A.D. Adler, F.R. Longo, F. Kampas, J. Kim, J. Inorg. Nucl. Chem. 32, 2443 (1970).

[10] K. Burda, A. Hrynkiewicz, H. Kołoczek, J. Stanek, K. Strzałka, Biochem. Biophys. Acta 1244, 345 (1995).

[11] K. Dziedzic-Kocurek, J. Stanek, J. Mag. Mag. Mater., submitted for publication.

[12] S.B. Brown, P. Jones, I.R. Lantzke, Nature 223, 960 (1969).

[13] R.M. Wing, K.P. Callahan, Inorg. Chem. 8, 871 (1969).

[14] K.S. Murray, Coord. Chem. Rev. 12, 1 (1974).

[15] D.M. Kurtz, Chem. Rev. 90, 585 (1990).

[16] E.B. Fleischer, T.S. Srivastava, J. Am. Chem. Soc. 91, 2403 (1969).

[17] J.O. Alben, in: The Porphyrins, Vol. III, Ed. D. Dolphin, Academic Press, New York 1978, p. 323.

[18] W.I. White, in: The Porphyrins, Vol. 5, Part C, Ed. D. Dolphin, Academic Press, New York 1978, p. 303.

[19] A.R. Li, H.H. Wei, L.L. Gang, Inorg. Chim. Acta 290, 51 (1999).

[20] X. Liu, E.K.L. Yeow, S. Velate, R.P. Steer, Phys. Chem. Chem. Phys. 8, 1298 (2006).

[21] M. Gouterman, in Ref. [17], p. 1.

[22] C.A. Brown, G.J. Remar, R.L. Musselman, E.I. Solmon, Inorg. Chem. 34, 688 (1995).

[23] M.W. Makinen, A.K. Churg, in: Iron Porphyrins, Part I, Eds. A.B.P. Lever, H.B. Gray, Addison-Wesley Publ. Co., Canada 1983, p. 141.

[24] A.K. Shiemke, T.M. Loehr, J. Sanders-Loehr, J. Am. Chem. Soc. 106, 4951 (1984).

[25] J. Sanders-Loehr, W.D. Wheeler, A.K. Shiemke, B.A. Averill, T.M. Loehr, J. Am. Chem. Soc. 111, 8084 (1989).

[26] R.H. Felton, N.T. Yu, in Ref. [17], p. 347.

[27] T.G. Spiro, in: Iron Porphyrins, Part II, Eds. A.B.P. Lever, H.B. Gray, Addison-Wesley Publ. Co., Canada 1983, p. 89.

[28] J.B. Vincent, G.L. Olivier-Lilley, B.A. Averill, Chem. Rev. 90, 1447 (1990). 